\title{
Oligomerization of esculin improves its antibacterial activity and modulates antibiotic resistance
}

\author{
Imen Mokdad-Bzeouich ${ }^{1,2}$, Nadia Mustapha ${ }^{1,2}$, Fadwa Chaabane ${ }^{1,2}$, Zied Ghedira ${ }^{1,2}$, Kamel Ghedira ${ }^{2}$, \\ Mohamed Ghoul ${ }^{3}$, Latifa Chebil ${ }^{3}$ and Leila Chekir-Ghedira ${ }^{1,2}$
}

In this particular study, the antibacterial activity of esculin and oligomer fractions was assessed. MIC values of esculin and its oligomer fractions as well as of some antibiotics against Gram-positive and Gram-negative strains and against Escherichia coli multiresistant variants were determined by the standard broth microdilution method. Both esculin and oligoesculin fractions exhibited antibacterial effect against reference strains; Staphylococcus aureus, Enterococcus faecalis, Salmonella enteritidis and Salmonella typhimurium. It appears that E3 oligomer fraction had the greatest antibacterial activity against these reference strains. Besides, as E2 and E3 revealed the best antibacterial effect against multiresistant variants of $E$. coli, we decided to test the effect of each, combined to the antibiotic against which the variants were resistant. In the interaction study, E2 and E3 oligoesculin fractions were found to be effective in reducing the resistance of $E$. coli 6574 to ofloxacin and the resistance of $E$. coli 6228 to amoxicillin. Only E3 oligoesculin fraction showed a synergetic interaction with amoxicillin and tetracyclin against $E$. coli 6708 , but no interaction was found either with E2 or E3 fractions against $E$. coli 6234 . Our study allowed us to conclude that oligomerization of esculin increases its antibacterial potential, according to the degree of polymerization. The Journal of Antibiotics (2015) 68, 148-152; doi:10.1038/ja.2014.127; published online 24 September 2014

\section{INTRODUCTION}

Escherichia coli is one of the most common bacteria of the microbial flora of gastrointestinal tract of poultry and human being, as well as other animals, but may become pathogenic to both. ${ }^{1,2}$ It is considered as one of the principal causes of infectious diseases in humans. This bacterium is known to produce enterotoxins involved in diarrheal diseases. The activity of cytotoxins and their role in human infection have been identified mainly in the infections of urinary tracts, meningitis, endocarditis, septicemia and epidemic diarrhea of adults and children. ${ }^{3-5}$ These microorganisms originating from food animals have been frequently developing resistance to many antibiotics, commonly used to control infectious diseases. Resistant organisms may be transferred to humans either directly, via the food chain, or indirectly as a result of the spread of animal waste in fields. ${ }^{6}$ In addition, antibiotics are sometimes associated with adverse effects, in the host, including hypersensitivity, depletion of gut and mucosal microorganisms, immunosuppression and allergic reactions. For patients, this antimicrobial resistance increases morbidity and mortality, and accordingly a significant increase in costs for health care. Therefore, there is a need of alternative antimicrobial drugs for the treatment of infectious diseases. One approach is to screen local medicinal plants for possible antimicrobial properties. These plants represent a rich source of novel antibacterial and antifungal compounds. ${ }^{7,8}$
Coumarins are found in many plant families, such as Apiaceae, Asteraceae, Fabiaceae, Rosaceae, Rubiaceae, Rutaceae and Solanaceae. ${ }^{9}$ The antibacterial properties of coumarins were first recognized in 1945 by Goth, ${ }^{10}$ who reported that dicoumarol inhibits the growth of several bacterial strains. The study of Melliou et al. ${ }^{11}$ revealed that coumarins have a broad spectrum of activity against Gram-positive and Gram-negative bacteria. Whereas, Evans et al. ${ }^{12}$ have shown that anthogenol, a coumarin compound from green fruits of Aegle marmelos, exhibits an antibacterial activity against Enterococcus strains. Another study conducted by Basile et al..$^{13}$ reported the efficacy of two coumarins aegelinol and agasyllin in inhibiting Helicobacter pylori growth in a dose-dependent manner (5 and $25 \mu \mathrm{g} \mathrm{ml}^{-1}$ ). Moreover, imperatorin a furancoiumarin isloated from Angelica dahurica and Angelica archangelica (Umbelliferae), respectively, shows antibacterial activity against Shigella dysenteriae. ${ }^{14,15}$

In this study we investigated the antimicrobial potential of a coumarin, which is esculin and its oligomers. Esculin is a monosaccharide of esculetin characterized by a fine blue fluorescent solution. It is extracted from the Aesculus hippocastanum L. (Hippocastanaceae) or horse-chestnut tree. In consideration of extension of the amplification of physicochemicals (solubility and thermal stability) and biological properties (antioxidant and antimutagenic) of the coumarins, as it was recently reported by Anthoni et al. ${ }^{16}$ and

${ }^{1}$ Laboratoire de Biologie Cellulaire et Moléculaire, Faculté de Médecine Dentaire, Université de Monastir, Rue Avicenne, Monastir, Tunisie; ${ }^{2}$ Unité de Substances Naturelles Bioactives et Biotechnologie UR12ES12, Faculté de Pharmacie de Monastir, Université de Monastir, Rue Avicenne, Monastir, Tunisie and ${ }^{3}$ Laboratoire d'Ingénierie des Biomolécules, ENSAIA-INPL, Université de Lorraine, Vandoeuvre-lès-Nancy, France

Correspondence: Professor L Chekir-Ghedira, Laboratoire de Biologie Cellulaire et Moléculaire, Faculté de Médecine Dentaire, Université de Monastir, Rue Avicenne, Monastir 5000, Tunisie.

E-mail: leila.chekir@laposte.net

Received 17 January 2014; revised 28 July 2014; accepted 21 August 2014; published online 24 September 2014 
Ben Rhouma et al., ${ }^{17}$ and since few studies have considered polymerization as a manner to improve physiochemical and biological properties of compounds, ${ }^{16,17}$ we attempted to study the oligomerization of esculin to ameliorate its properties. Polymerization reaction of esculin is catalyzed by laccase enzyme and carried out in mild conditions of $\mathrm{pH}$, temperature and pressure to maintain stability of molecules and to allow the synthesis of conjugated polymers in continuous without using toxic reagents. ${ }^{18}$ The aim of this study was to investigate the effectiveness of esculin and its oligomers against Gram-positive and Gram-negative bacteria, as well as against four multiresistant variants of $E$. coli and their modulating effect on bacterial resistance to the target antibiotics.

\section{MATERIALS AND METHODS}

\section{Bacterial material}

Five bacterial species, Staphylococcus aureus ATCC 25923, Enterococcus faecalis ATCC 29212 (Gram positive), Escherichia coli ATCC 25922, Salmonella enteritidis ATCC 13076, Salmonella typhimurium NRRLB 4420 (Gram negative) were used as reference strains.

Synergetic activities of the molecules were also tested against four multidrugresistant variants of E. coli with the resistance profile described in Table 1. All these variants were kindly provided from the clinical bacterial collection of CHU Fattouma Bourguiba, Monastir-Tunisia. The cultures were grown in nutrient broth (Sigma-Aldrich, Canada Ltd, Oakville, ON, Canada).

\section{Antimicrobial agents}

The antibiotics used were tetracyclin, amoxicillin, ofloxacin, cefotaxim and piperacillin (Sigma-Aldrich, Canada Ltd).

\section{Oligomerization reaction and characterization of oligoesculin fractions}

The molecules used against the genera of bacterial pathogens were esculin and its oligomers obtained as previously described by Ben Rhouma et al. ${ }^{17}$ Briefly, esculin $\left(50 \mathrm{gl}^{-1}\right)$ was suspended in 1 liter of a methanol/water $(30: 70 \mathrm{v} / \mathrm{v})$ reaction medium. Laccase solution $\left(3 \mathrm{U} \mathrm{ml}^{-1}\right)$ was added to the mixture. The reaction was stirred at 600 r.p.m., for $72 \mathrm{~h}$, at $20^{\circ} \mathrm{C}$. The kinetics of polymerization reaction were followed by size exclusion chromatography. The final reaction media, enriched with esculin oligomers, was separated, by successive filtration processes, on a $15 \mathrm{kDa}$ membrane, to remove the enzyme, and then successively on 5, 3 and $1 \mathrm{kDa}$ membranes by a diafiltration process (INSIDE CeRAM, Nyons, France), using a mixture of water/methanol (70:30 v/ v) (5 liters) as eluent, at $50{ }^{\circ} \mathrm{C}$.Three oligomer fractions of esculin: E1 (retentate obtained after diafiltration of oligoesculin on a $1 \mathrm{kDa}$ membrane), E2 (retentate obtained after diafiltration of oligoesculin on a $3 \mathrm{kDa}$ membrane) and $\mathrm{E} 3$ (retentate obtained after diafiltration of oligoesculin on a $5 \mathrm{kDa}$ membrane) were obtained. These fractions were lyophilized (Bioblock lyophilizer, Bioblock Scientific, Illkirch, France) and characterized (Table 2). Relative masses of

Table 1 Antibiotic resistance profile of $E$. coli variants

\begin{tabular}{|c|c|}
\hline Bacteria strains & Antibiotic resistance \\
\hline \multicolumn{2}{|l|}{ E. coli } \\
\hline 6574: cephalosporinase & $\begin{array}{l}\text { AMX, TIC, CAZ, TIC+CLA, PIP,CTX,CEF, AMX } \\
+ \text { CLA, FAM, FOX, RIF, OFX, TET, AMK }\end{array}$ \\
\hline 6228: high level penicillinase & AMX, TIC, PIP, CLA+AMX, TIC+CLA \\
\hline 6708: low level penicillinase & AMX, TIC, TET, MEC \\
\hline $\begin{array}{l}\text { 6234: extended-spectrum } \\
\beta \text {-Lactamase }\end{array}$ & $\begin{array}{l}\text { AMX, TIC, CEF, PIP, CTX, FPO, CEF, FAM, } \\
\text { GEN,TOB,TET,CIP,OFX, NOR, RIF, NIT, NAL }\end{array}$ \\
\hline \multicolumn{2}{|c|}{$\begin{array}{l}\text { Abbreviations: AMK, amikacine; AMX, amoxicillin; CAZ, ceftazidime; CEF, cephalothin; CIP, } \\
\text { ciprofloxacin; CLA, clavulin; CTX, cefotaxim; FAM, cefamandole; FOX, cefoxitin; FPO, cefpirom; } \\
\text { GEN, gentamicin; MEC, mecillinam; NAL, nalidixic acid; NIT, nitrofurantoin; NOR, norfloxacin; } \\
\text { OFX, ofloxacin; PIP, piperacillin; RIF, rifampicin; TET, tetracyclin; TIC, ticarcillin; TOB, } \\
\text { tobramycin. }\end{array}$} \\
\hline
\end{tabular}

polymers were evaluated by size exclusion chromatography (HPLC LaChrom, UV $280 \mathrm{~nm}$ LaChrom L-7400, Tosoh TSKgel $\alpha 3000$ column, $\left.60{ }^{\circ} \mathrm{C}\right) .{ }^{19}$ The obtained data allowed the determination of number-average molecular mass, weight-average molecular mass, weight-average-molecular mass index and polydispersity:

$$
\begin{aligned}
& \bar{M}_{\mathrm{n}}=\frac{\sum_{i} n_{i} M_{i}}{\sum_{i} n_{i}}: \text { Number-average molecular mass } \\
& \bar{M}_{\mathrm{w}}=\frac{\sum_{i} w_{i} M_{i}}{\sum_{i} w_{i}}: \text { Weight-average molecular mass } \\
& I_{\mathrm{M}}=\frac{\bar{M}_{\mathrm{w}}}{M_{1}}: \text { Molecular mass index } \\
& \text { PDI }=\frac{\bar{M}_{\mathrm{w}}}{\bar{M}_{\mathrm{n}}}: \text { Polydispersity }
\end{aligned}
$$

\section{MIC determination of molecules and antibiotics}

The resistance of bacteria to the antibiotics and the MIC of natural antimicrobials were determined by the standard broth microdilution method. ${ }^{20}$ One hundred microliters of an overnight bacterial culture (cultivated for $16 \mathrm{~h}$ at $37^{\circ} \mathrm{C}$ ) diluted to a cell density of $10^{6} \mathrm{cfu} \mathrm{ml}^{-1}$ and $100 \mu \mathrm{l}$ of the tested compounds (concentrations obtained by serial dilution; 5000, 2500, $1250,625,312$ and $156 \mathrm{mgl}^{-1}$ ) or of the tested antibiotics were seeded in 96well microtitration plates and incubated overnight at $37^{\circ} \mathrm{C}$. Twenty-four hours after seeding, $40 \mu \mathrm{l}$ of 2-[4-iodophenyl]-3-[4-dinitrophenyl]-5-phenyltetrazolium chloride (Sigma, St Louis, MO, USA) were added to each well, and plates were incubated for 1 to $2 \mathrm{~h}$ at $37^{\circ} \mathrm{C} .{ }^{21}$ The electron transport system of respiring organisms reduces 2-[4-iodophenyl]-3-[4-dinitrophenyl]-5-phenyltetrazolium chloride to 2-[4-iodophenyl]-3-[4-dinitrophenyl]-5-phenyltetrazolium chloride-formazan with a purple color. The MIC was considered as the lowest concentration of the tested compound that prevented the formation of such color. Determination of MIC values was made in triplicate.

\section{Synergistic activity of oligoesculin fractions and antibiotics}

The effect of combination of oligomer fractions (E2 and E3) with antibiotics on bacterial growth in liquid suspension was determined using the standard broth microdilution assay. Overnight cultures of strains were diluted to $10^{6} \mathrm{cfu} \mathrm{ml}^{-1}$ in nutrient broth and were incubated in a 96-well microtitration plates. Concentrations corresponding to the MIC value of each natural compound and that of the antibiotic to which the tested variant of E. coli was resistant, were combined and added to the microtiter plate wells. Then, twofold serial dilutions of the combination mixture were performed (MIC/2, MIC/4, MIC/8 and MIC/ 16). The resulting inhibitory concentrations were used to calculate the FIC (fractional inhibitory concentration) which is the sum of FICA and FICB, where FICA is the MIC of the compound in combination with the antibiotic/ MIC of the compound alone, and FICB is the MIC of the antibiotic in combination with the tested compound/MIC of the antibiotic alone. FIC values

Table 2 Weight-average-molecular mass index, polydispersity and weight-average-molecular mass of oligoesculin fractions

\begin{tabular}{lccc}
\hline Fractions & $\bar{M}_{\mathrm{w}}\left(\mathrm{g} \mathrm{mol}^{-1}\right)$ & $\mathrm{PDI}$ & $\mathrm{I}_{M}$ \\
\hline Esculin & 339.36 & 1.00 & 1.00 \\
E1 & 1021.33 & 1.48 & 1.48 \\
E2 & 3042.10 & 1.39 & 1.39 \\
E3 & 5080.43 & 1.41 & 1.41 \\
\hline
\end{tabular}

Abbreviations: $\mathrm{E} 1$, retentate obtained after diafiltration of oligoesculin on a $1 \mathrm{kDa}$ membrane; $\mathrm{E} 2$, retentate obtained after diafiltration of oligoesculin on a $3 \mathrm{kDa}$ membrane; E3, retentate obtained after diafiltration of oligoesculin on a $5 \mathrm{kDa}$ membrane; $I_{\mathrm{M}}$, weight-average-molecular mass index; $\bar{M}_{\mathrm{w}}$, weight-average molecular mass; PDI, polydispersity. 
were interpreted as follows; FICA+FICB $\leqslant 0.5$ means a synergistic effect, FICA + FICB comprised between 0.5 and 4 , means no interaction and FICA+FICB $>4$, means an antagonist effect. ${ }^{21}$ The experiments were made in triplicate.

\section{RESULTS}

The antimicrobial activity of the molecules was evaluated against five reference bacteria. It appears that esculin and its oligomer fractions exhibited various levels of antibacterial effects against the tested bacterial strains. MIC values varied from 312 to $5000 \mathrm{mgl}^{-1}$. The results summarized in Table 3 showed that oligoesculin fractions were more effective in reducing the growth of bacteria compared with monomeric esculin. S. enteritidis is the most susceptible bacterial species to esculin, E1 and E2 fractions with the same MIC values of $625 \mathrm{mg} \mathrm{l}^{-1}$. Moreover, E1 and E2 were efficient against $S$. aureus with MIC values of 1250 and $625 \mathrm{mg} \mathrm{l}^{-1}$, respectively. E3 fraction displayed strong activity against both Gram-negative and Gram-positive bacteria as it gives the lowest MIC values with all the tested bacteria. Indeed, E. coli and S. typhimurim were the most susceptible bacterial species to $\mathrm{E} 3$ fraction with a MIC value of $312 \mathrm{mg}^{-1}$. The antimicrobial activity of the tested molecules was also evaluated against four multidrugresistant variants of $E$. coli. Their resistance profiles are described in Table 1. Results in Table 3 showed that esculin and its oligomer fractions were efficient toward these variants, with MIC values varying from 312 to $2500 \mathrm{mg} \mathrm{l}^{-1}$.

It was found that oligomers E1, E2 and E3 displayed inhibiting activity against resistant variants of $E$. coli in comparison with the monomeric esculin. E. coli 6574 was the most susceptible bacteria to E1 fraction with a MIC value of $625 \mathrm{mgl}^{-1}$, followed by E. coli 6234, with a MIC value of $1250 \mathrm{mg} \mathrm{l}^{-1}$, then both E. coli 6228 and 6708 with a MIC value of $2500 \mathrm{mg} \mathrm{l}^{-1}$. E2 fraction exhibited the same effect as E1 toward the four tested variants, except against E. coli 6708 which revealed a MIC value of $625 \mathrm{mg} \mathrm{l}^{-1}$. E3 was the most efficient fraction in inhibiting multiresistant variants of $E$. coli growth, with MIC values ranging from 312 to $625 \mathrm{mg} \mathrm{l}^{-1}$.

As far as E2 and E3 exhibited the most antibacterial activity against the tested variants, we attempted to study their potential to modulate the resistance of the tested variants toward the corresponding antibiotics. At first, we determined the new inhibitive minimal concentrations of antibiotics to which E. coli variants were resistant, and then we looked for the presence or not of a synergistic effect between the tested fractions and each of these antibiotics, in order to detect whether fractions could restore the sensibility of bacteria to these antibiotics or not.
Our results revealed that E2 and E3 fractions have a synergistic interaction with ofloxacin against E. coli 6574 , as assessed by FIC values $(\mathrm{FIC}=0.5)$, as far as MIC value of ofloxacin was reduced from $250 \mathrm{mgl}^{-1}$ (in the absence of oligomer fractions) to $62 \mathrm{mgl}^{-1}$ (in the presence of oligomer fractions) (Table 4). However, no interaction was observed with cefotaxim $(\mathrm{FIC}=1)$. Synergistic interaction was observed also between each of E2 and E3 oligomer fractions and amoxicillin against E. coli 6228. In fact, both E2 and E3 fractions in combination with amoxicillin reduced its MIC value from 1250 $\mathrm{mgl}^{-1}$ to $78 \mathrm{mgl}^{-1}$ (Table 4). Likewise, E3 oligomer fraction exhibited a synergetic interaction with both amoxicillin and tetracyclin against $E$. coli 6708, as MIC values of the tested antibiotics decreased from 1250 to $312 \mathrm{mgl}^{-1}$ and from 125 to $31 \mathrm{mgl}^{-1}$, respectively (Table 4). Whereas no interaction was detected between the tested fractions and cefotaxim or ofloxacin, against E. coli 6234, FIC index value was $>0.5$ (Table 4 ).

\section{DISCUSSION}

In the present study, esculin and its oligomer fractions (E1, E2 and E3) were tested for their antibacterial activity against five strains of bacteria: two Gram-positive bacteria ( $S$. aureus and E. faecalis) and three Gram-negative bacteria (E. coli, S. typhimurium and S. enteritidis), as well as against four multiresistant variants of $E$. coli. Bacterial susceptibility to esculin and its oligomer fractions was evaluated by determining the MIC. Each compound showed more or less pronounced antibacterial potencies, affecting both Gram-positive and Gram-negative bacteria.

Gram-negative bacteria differs from Gram-positive bacteria by the presence of an outer membrane and unique periplasmic space, which is not found in Gram-positive bacteria. The resistance of Gramnegative bacteria toward antibacterial substances is related to the hydrophilic surface of their outer membrane which is rich in lipopolysaccharide molecules, presenting a barrier to the penetration of numerous antibiotic molecules, and is also associated with the enzymes in periplasmic space, which are capable of breaking down the molecules introduced from outside. ${ }^{22}$ It appears from previous studies that oligomerization of esculin ameliorates its solubility, ${ }^{16}$ and it could facilitate its passage through the outer membrane of Gram-negative bacteria making them so effective against Gram-positive as against Gram-negative bacteria.

It was shown that oligomer fractions exhibited better antibacterial activity than the monomeric esculin. Oligoesculin fraction E3 induced

Table 3 Antimicrobial activity of esculin and its oligomer fractions, expressed as MICa $\left(\mathrm{mg} \mathrm{I}^{-1}\right)$

Escherichia coli multiresistant

References strains variants

\begin{tabular}{|c|c|c|c|c|c|c|c|c|}
\hline \multicolumn{2}{|c|}{ Gram-positive } & \multicolumn{3}{|c|}{ Gram-negative } & \multirow{2}{*}{6574} & \multirow{2}{*}{6228} & \multirow{2}{*}{6708} & \multirow{2}{*}{6234} \\
\hline Staphylococcus aureus & Enterococcus faecalis & Escherichia coli & Salmonella enteritidis & Salmonella typhimurium & & & & \\
\hline 2500 & 2500 & 2500 & 625 & 5000 & 1250 & 2500 & 2500 & 1250 \\
\hline 1250 & 2500 & 2500 & 625 & 2500 & 625 & 2500 & 2500 & 1250 \\
\hline 625 & 2500 & 1250 & 625 & 1250 & 625 & 2500 & 625 & 1250 \\
\hline 625 & 625 & 312 & 625 & 312 & 625 & 312 & 312 & 312 \\
\hline
\end{tabular}

Abbreviations: E1, retentate obtained after diafiltration of oligoesculin on a $1 \mathrm{kDa}$ membrane; $\mathrm{E2}$, retentate obtained after diafiltration of oligoesculin on a $3 \mathrm{kDa}$ membrane; E3, retentate obtained after diafiltration of oligoesculin on a $5 \mathrm{kDa}$ membrane. 6228 , E. coli high level penicillinase; $6234, E$. coli extended-spectrum $\beta$-lactamase; 6574, E. coli cephalosporinase; 6708 , E. coli low level penicillinase.

a MIC value \pm 0.00 (s.d.) $\left(\mathrm{mgl}^{-1}\right)$, means of three experiments. 
Table 4 Interaction between oligoesculin fractions (E2 and E3) and antibiotics and their effect against multiresistant variants of $E$. coli

\begin{tabular}{|c|c|c|c|c|c|c|c|c|}
\hline \multirow[b]{2}{*}{ E.coli variants } & \multirow[b]{2}{*}{ Agent } & \multicolumn{2}{|c|}{$\operatorname{MIC}\left(m g l^{-1}\right)$} & \multirow[b]{2}{*}{ FIC } & \multirow[b]{2}{*}{ Agent } & \multicolumn{2}{|c|}{$\operatorname{MIC}^{\mathrm{a}}\left(m g l^{-1}\right)$} & \multirow[b]{2}{*}{ FIC } \\
\hline & & Alone & Combined & & & Alone & Combined & \\
\hline \multirow{3}{*}{6574} & E2 & 625 & 312 & & E3 & 625 & 312 & \\
\hline & OFX & 250 & 62 & 0.5 & OFX & 250 & 62 & 0.5 \\
\hline & E2 & 625 & 156 & & E3 & 625 & 156 & \\
\hline \multirow{2}{*}{6228} & PIP & 250 & 125 & 1 & PIP & 250 & 125 & 1 \\
\hline & E2 & 2500 & 1250 & & E3 & 312 & 156 & \\
\hline \multirow[t]{4}{*}{6708} & AMX & 1250 & 1250 & 2 & AMX & 1250 & 312 & 0.5 \\
\hline & E2 & 625 & 625 & & E3 & 312 & 78 & \\
\hline & TET & 125 & 125 & 2 & TET & 125 & 31 & 0.5 \\
\hline & E2 & 625 & 625 & & E3 & 312 & 78 & \\
\hline
\end{tabular}

Abbreviations: AMX, Amoxicillin; CTX, Cefotaxim; E2, retentate obtained after diafiltration of oligoesculin on a $3 \mathrm{kDa}$ membrane; E3, retentate obtained after diafiltration of oligoesculin on a $5 \mathrm{kDa}$ membrane; OFX, Ofloxacin; PIP, Piperacillin; TET, Tetracyclin; 6228, E. coli high level penicillinase; 6234, E. coli extended-spectrum $\beta$-lactamase.; 6574 , E. coli cephalosporinase; 6708, E. coli low level penicillinase

a MIC value \pm 0.00 (s.d.) ( $\left.\mathrm{mg} \mathrm{I}^{-1}\right)$, means of three experiments.

the greatest antibacterial effect. Indeed, the antibacterial activity of oligomer fractions increased according to the degree of oligomerization. This is in a good accordance with results obtained by Hodák et al., ${ }^{23}$ who reported that coumarins having long chain hydrocarbon substitutions such as ammoresinol and ostruthin exhibited antibacterial activity against a wide spectrum of Gram-positive bacteria such as Bacillus megaterium, Micrococcus luteus and S. aureus. It was also reported that some coumarins such as aegelinol and agasyllin showed significant antibacterial activity against clinically isolated Grampositive and Gram-negative bacterial strains such as $S$. aureus, Salmonella typhimurium, Enterobacter cloacae and Enterococcus aerogenes. ${ }^{13}$ Moreover, Coumermycin, a natural coumarin that is structurally similar to novobiocin, exhibited a potent antibacterial activity against $E$. coli by inhibiting the supercoiling of DNA catalyzed by E. coli DNA gyrase. ${ }^{24}$ Another study conducted by De Souza et al. ${ }^{25}$ described the antibacterial activity of 45 coumarins against four bacterial strains: two Gram-positive bacteria ( $S$. and B. cereus) and two Gram-negative bacteria (E. coli and P. aeruginosa). The authors reported that the presence of $\mathrm{OH}$ at position 7 of the benzenic ring of coumarins, is required for their antibacterial activity against Grampositive bacteria. Similarly, the addition of ortho $\mathrm{OH}$ functions at C6 and C7 of coumarin ring (esculetin), displayed fairly high antibacterial activity, which is probably due to the facilitated interaction with the peptidoglycan found in the cell wall of Gram-positive bacteria. In addition, Kayser and Kolodziej ${ }^{26}$ have shown that, among 14 oxygenated coumarins, the 7-hydroxy-5, 6-dimethoxycoumarin present the most potent antimicrobial activity against eight different microorganisms. It seems according to these observations that the hydroxyl group in position 7 of esculin is involved in its antibacterial potential, and thus it should not be affected during the oligomerization reaction giving rise to oligoesculin fractions (Ben Rhouma et al. ${ }^{17}$ ).

Our estimation of the antimicrobial activity of esculin and oligoesculin fractions against the multidrug-resistant variants of E. coli revealed that E2 and E3 displayed a stronger activity than the monomer. They also modulate the resistance of the tested variants to antibiotics, when combined with them, by reducing significantly the MIC values of these antibiotics. E2 and E3 oligoesculin fractions, exhibited a synergistic interaction with ofloxacin and amoxicillin against E. coli 6574 and E. coli 6228, respectively. We noted a synergistic combination between E3 and amoxicillin, and E3 and tetracyclin against E. coli 6708. This is in accordance with the study of Smyth et al., ${ }^{27}$ who alluded that many natural and synthetic coumarins may modulate the resistance of methicillin-resistant S. aureus strains). For example, 8-Iodo-5, 7-dihydroxycoumarin displayed a more pronounced bioactivity in combination with oxacillin and vancomycin.

To date, only a limited number of coumarins have been assessed against the multidrug-resistant bacteria and the exact mechanism of the reduction of antimicrobial resistance by natural antimicrobials is unknown, but it is probably attributed to a structural change in the resistant bacteria. Reducing bacterial resistance to a given antibiotic may be related to a better penetration of the antibiotic through the outer layer of the bacterial cell wall, or the inhibition of some bacterial enzymes that confer protection to the strains. ${ }^{28}$ In addition, some compounds either natural or synthetic, modulate the resistance of bacteria via the elimination of plasmids that confer resistance to antibiotics, or by inhibition of the membrane efflux pump. In this case, an antibiotic that has a poor efficacy against a resistant strain can accumulate inside the bacterial cell and therefore have a greater antimicrobial effect. ${ }^{29,30}$

The present study demonstrates that esculin and its oligomer fractions were active against various bacterial strains. E2 and E3 fractions have synergistic interactions against multiresistant variants of E. coli. The use of these combinations is likely to reduce the MIC of antibiotics, thus minimizing their possible toxic side effects and their treatment cost. However, in order to assess the potential of these combinations for therapeutic application, further works are necessary to explore the mode of action of oligoesculin fractions and 
to understand the molecular mechanisms of their synergy with antibiotics.

\section{CONFLICT OF INTEREST}

The authors declare no conflict of interest.

\section{ACKNOWLEDGEMENTS}

We acknowldge the 'Ministère Tunisien de l'enseignement supérieur et de la recherche scientifique' and the 'Ministère français des Affaires Etrangères (Action Intégrée de Coopération Inter-universitaire Franco-Tunisienne, CMCU $11 \mathrm{G} / 118)^{\prime}$ for the financial support of this study.

1 Jawetz, E., Melnick, J. \& Adelberg, E. A. in Review of Medical Microbiology, 122-124 (Long Medical Publication: Los Altos, CA, 1984).

2 Levine, M. Escherichia coli that cause diarrhea: enterotoxigenic, enteropathogenic enteroinvasive, enterohemorrhagic and enteroadherent. J. Infect. Dis. 155 377-390 (1987).

3 Scotland, S. M., Day, N. P., Willshaw, G. A. \& Rowe, B. Cytotoxic enteropathogenic Escherichia coli. Lancet 1, 90 (1980)

4 Hughes, C., Muller, D., Hacher, J. \& Goebel, W. Genetics and pathogenic role of Escherichia coli haemolysin. Toxicon. 20, 247-252 (1982).

5 Daini, O. A, Ogbulo, O. D. \& Ogunledun, A. Quinolones Resistance and R-plasmids of some gram negative enteric Bacilli. AJCEM 6, 14-19 (2005).

6 Hammerum, A. M. \& Heuer, O. E. Human health hazards from antimicrobial resistant Escherichia coli of animal origin. Clin. Infect. Dis. 48, 916-921 (2009).

7 Rates, S. M. K. Plants as source of drugs. Toxicon. 39, 603-613 (2001)

8 Mahesh, B. \& Satish, S. Antimicrobial activity of some important medicinal plant against plant and human pathogens. World. J. Agric. Sci. 4, 839-843 (2008)

9 Weinmann, I. in Biology, Applications and Mode of Action. History of The Development and Applications of Coumarin and Coumarin-Related Compounds (eds O'Kennedy, R. Thornes, R.D.) 1-22 (John Wiley \& Sons, Inc.: New York, NY, 1997).

10 Goth, A. The antibacterial properties of dicumarol. Science 101, 384 (1945).

11 Melliou, E. et al. Natural and synthetic 2, 2-dimethylpyranocoumarins with antibacterial activity. J .Nat. Prod. 68, 78-82. (2005).

12 Evans, W. C. Trease and Evans Pharmacognosy 16th edn (Elsevier Ltd., 2009).

13 Basile, A. et al. Antimicrobial and antioxidant activities of coumarins from the roots of Ferulago campestris (Apiaceae). Molecules 14, 939-952 (2009).

14 Baek, N., Ahn, E. M, Kim, H. Y. \& Park, Y. D. Furanocoumarins from the root of Angelica dahurica. Arch. Pharm. Res. 23, 467-470 (2000).
15 Raja, S. B., Murali, M. R., Roopa, K. \& Devaraj, S. N. Imperatorin a furocoumarin inhibits periplasmic Cu-Zn SOD of Shigella dysenteriae their by modulates its resistance towards phagocytosis during host pathogen interaction. Biomed. Pharmacother. 65 560-568 (2011)

16 Anthoni, J. et al. Enzymatic synthesis of oligoesculin: structure and biological activities characterizations. Eur. Food Res. Technol. 231, 571-579 (2010).

17 Ben Rhouma, G., Chebil, L., Krifa, M., Ghoul, M. \& Chekir-Ghedira, L. Evaluation of mutagenic and antimutagenic activities of oligorutin and oligoesculin. Food Chem. 135 1700-1707 (2012).

18 Kobayashi, S., Uyama, H. \& Kimura, S. Enzymatic Polymerization. Chem. Rev. 101, 3793-3818 (2001)

19 Faix, O., Lange, W. \& Salud, E. C. The use of HPLC for the determination of average molecular weights and molecular weight distributions of milled wood lignins from Shorea polysperma (Blco.). Holzforschung 35, 3-9 (1981).

20 Wiegand, I., Hilpert, K. \& Hancock, R. E. W. Agar and broth microdilution methods to determine the minimal inhibitory concentration (MIC) of antimicrobial substances. Nat Protoc. 3, 163-175 (2008).

21 Palaniappan, K. \& Holley, R. A. Use of natural antimicrobials to increase antibiotic susceptibility of drug resistant bacteria. Int. J. Food Microbiol. 140 164-168 (2010).

22 Gao, Y., Belkum, M. J. V. \& Stiles, M. The outer membrane of Gram-negative bacteria inhibits antibacterial activity of Brochocin C. Applied Environ. Microbiol. 65, 4329-4333 (1999).

23 Hodák, K. Jakesová, V. \& Dadák, V. On the antibiotic effects of natural coumarins. VI. The relation of structure to the antibacterial effects of some natural coumarins and the neutralization of such effects. CeskaSlov. Farm 16, 86-91 (1967).

24 Gellert, M., O'Dea, M. H., Itoh, T. \& Tomizawa, J. I. Novobiocin and coumermycin inhibit DNA supercoiling catalyzed by DNA gyrase. Proc. Natl Acad. Sci. USA. 73 4474-4478 (1976)

25 De Souza, S. M., Delle Monache, F. \& Smânia, A. Jr. Antibacterial Activity of Coumarins. Z. Naturforsch. 60, 693-700 (2005).

26 Kayser, O. \& Kolodziej, H. Antibacterial activity of simple coumarins structural requirements for biological activity. Z. Naturforsch. 54, 169-174 (1999).

27 Smyth, T., Ramachandran, V. N. \& Smyth, W. F. A study of the antimicrobial activity of selected naturally occurring and synthetic coumarins. Int. J. Antimicrob. Ag. 33 421-426 (2008).

28 Darwish, R. M, Aburjai, T., Al-Khalil, S. \& Mahafzah, A. Screening of antibiotic resistant inhibitors from local plant materials against two different strains of Staphylococcus aureus. J. Ethnopharmacol. 79, 359-364 (2002)

29 Coutinho, H. D. M., Costa, J. G. M., Lima, E. O., Falcão-Silva, V. S. \& Siqueira, J. P. Herbal therapy associated with antibiotic therapy: potentiation of the antibiotic activity against methicillin-resistant Staphylococcus aureus by Turnera ulmifolia L. BMC Complement Altern. Med. 9, 13 (2009)

30 Rodrigues, F. F. G., Costa, J. G. M. \& Coutinho, H. D. M. Synergy effects of the antibiotics gentamicin and the essential oil of Croton zehntneri. Phytomedicine 16, 1052-1055 (2009). 